\title{
Visualizing the Development of Tissue Engineered Bone Constructs with a Low Field Benchtop MRI
}

\author{
Huihui XU \\ School of Engineering and Computer Science \\ University of the Pacific \\ Stockton, CA, USA \\ e-mail: hxu@pacific.edu
}

\begin{abstract}
This paper presents the possibility of utilizing a low field benchtop magnetic resonance imaging (MRI) to visualize the development of mesenchymal stem cells (MSCs) based tissue engineered (TE) bone constructs over a four-week period. All MRI measurements were conducted using a 0.5T MRI tomography system. Preliminary results demonstrated that the proposed approach is promising in differentiating various growth stages in engineered bone constructs non-constructively.
\end{abstract}

Keywords-Low Field Magnetic Resonance Imaging (MRI); Tissue Engineered (TE) Bone; Mesenchymal Stem Cells (MSCs); Tissue Development

\section{INTRODUCTION}

Natural bone healing following injury or disease is the preferred option to overcome osteogenic tissue loss and bone damage. But because bone has a limited capability to regenerate and remodel surgeons often need to implant synthetic materials or bone grafts at the site of injury. Approximately one million cases of bone grafting are performed annually in the U.S. to treat nonunion following fracture, at an estimated cost of over three billion dollars per year ${ }^{[1]}$. Both autologous and allogeneic bone grafts are used clinically as bone substitutes. The availability of compatible grafts is limited, however, because harvesting bone is painful and the procedures carry a high risk of infection. Therefore, tissue engineering is a progressively promising alternative currently, there are more than 10 clinical reports applying cell-based TE grafts for bone regeneration ${ }^{[2]}$. The success of TE requires gradual assessment of the outcome of developing tissues. Different growth stages of mesenchymally derived constructs are defined by changes in the construct phenotype (e.g. cell proliferation followed by extracellular matrix development and finally bone mineralization). This progression is conventionally characterized by destructive gene expressions at each stage ${ }^{[3]}$. The potential of non-invasive medical imaging techniques in monitoring developing tissues has recently been recognized. In particular, MRI could be used to visualize structural and functional changes associated with TE formation ${ }^{[4-7]}$.

\section{MATERIALS AND METHODS}

Healthy human bone MSCs isolated from fresh marrow cells were acquired commercially (Lonza, Walkersville, MD, USA). Upon 80 percent confluence, the MSCs were trypsinized, counted, and passaged at a density of $5 \times 103$ cells $/ \mathrm{cm} 2$ and expanded in vitro. Silk scaffolds (obtained from our collaborator in Tufts University) were biopsy punched with a 5-mm diameter and cut to a 5-mm thickness and then autoclaved in distilled water for a liquid cycle for 20 minutes before being removed and placed in basic media for conditioning. Scaffolds were seeded at 106 cells/scaffold, which were delivered in equal portions to the top and bottom surfaces. Osteogenic medium was used to induce osteogenesis in the constructs and at various growth stages samples were fixed in formalin for MRI.

All MRI experiments were conducted using a benchtop MRI tomography (Magritek, Aachen, Germany). The system has a field strength of $0.5 \mathrm{~T}$ with a $5-\mathrm{cm}$ radiofrequency (RF) solenoid coil with triple axis gradients (maximum strength of $0.25 \mathrm{~T} / \mathrm{m}$ ) allowing isotropic resolution up to $150 \mu \mathrm{m}$ with chemical resolution $<1 \mathrm{ppm}$. Spin-echo imaging sequence was used to acquire 64 echoes with a repetition time of 4000 msec and eight msec echo spacing from the chosen axial slice.

\section{RESULTS}

Fig. 1 shows an MR image acquired for four samples simultaneously presenting four different growth stages of TE bone constructs week1-4. A decrease in image intensity is visible with tissue development, which matches previous findings measured at different magnetic strength [6, 7]. Primary reason for the reduced intensity is, with the progression of osteogenic differentiation, i.e., the development of bone constructs, the bulk water concentration decreases as water molecules become immobilized. Meanwhile, mineral deposition in the extracellular matrix of TE constructs establishes local regions of solid bone-like components that introduce magnetic susceptibility variation, which results in a reduction in the $\mathrm{T} 2$. 


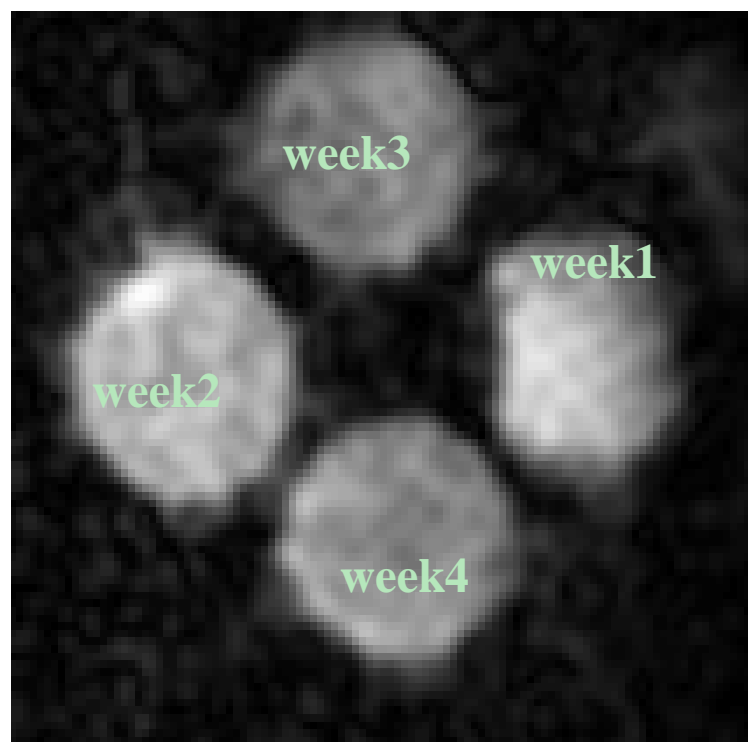

Figure 1. Preliminary MRI result of TE bone constructs at week1-4. Spin echo imaging sequence was used with acquisition parameters as follows: field of view $=35 \times 35 \mathrm{~mm}$; matrix size $=128 \times 128$ pixels; slice thickness $=5 \mathrm{~mm} ; \mathrm{TR}=4000 \mathrm{msec} ;$ effective $\mathrm{TE}=10 \mathrm{msec} ;$ number of echoes $=64 ; \mathrm{NEX}=128 ;$ spatial resolution is 312 microns.

It is also worth mentioning that, the use of a low magnetic field makes it more feasible to image engineered bone tissues with relative low $\mathrm{T} 2$ relaxation time; which was an issue in studies with higher magnetic fields because the $\mathrm{T} 2$ relaxation time is significantly reduced with the increased magnetic field strength compromising MR images. Below is a figure showing TE bone MRI with an echo time up to $180 \mathrm{msec}$.

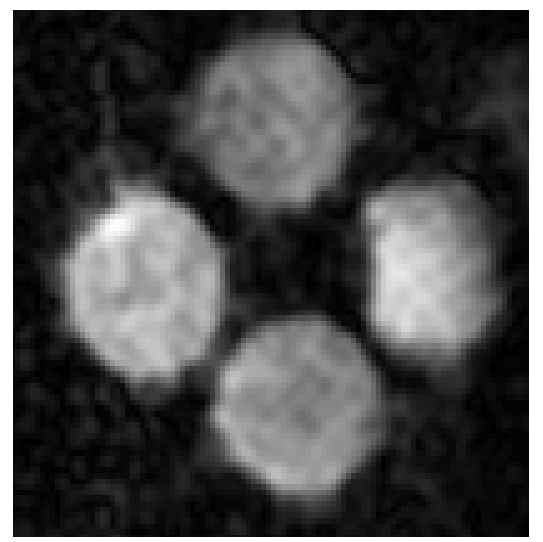

$\mathrm{TE}=7 \mathrm{msec}$

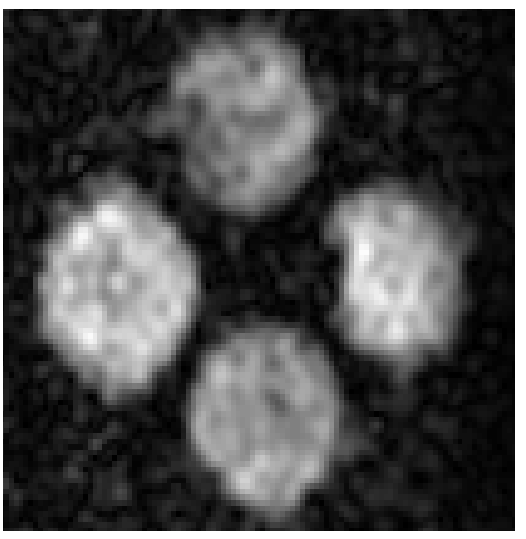

$\mathrm{TE}=80 \mathrm{msec}$

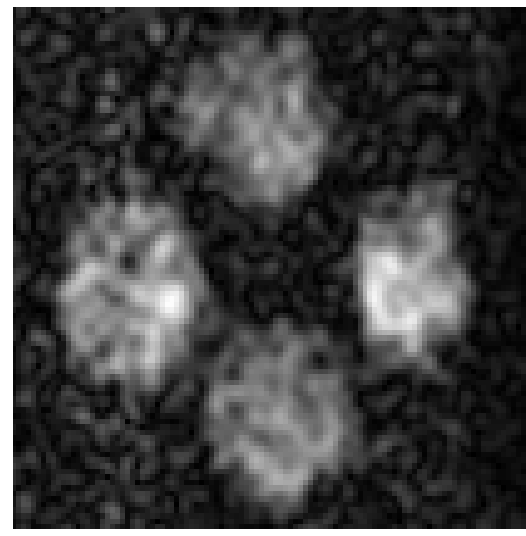

$\mathrm{TE}=180 \mathrm{msec}$

Figure 2. MRI result of TE bone constructs acquired with various echo time, up to $180 \mathrm{msec}$. Other imaging acquisition parameters are omitted.

\section{CONCLUSION}

TE constructs undergoing osteogenesis has known to exhibit a significant change in the relaxation times and other measurable properties. This preliminary study performed at low magnetic field $(0.5 \mathrm{~T})$ indicated the possibility of utilizing low field MRIs to visualize the development of osteogenesis in TE bone constructs. Low field MRIs thus appear to be a valuable tool for characterizing the early stages of osteogenesis and monitoring tissue regeneration in tissue engineering. This study focused on in vitro tissue development; in future such studies could be extended to investigate in vivo osteogenic tissue regeneration.

\section{ACKNOWLEDGEMENT}

The author would like to thank Dr. David L. Kaplan, Stern Family Professor and Chair of Biomedical Engineering at Tufts University, for providing the silk scaffold for the preparation of the TE constructs.

\section{REFERENCES}

[1] Laurencin, C. T. et al. The ABJS Nicolas Andry Award: Tissue engineering of bone and ligament: a 15-year perspective. Clin Orthop Relat Res 447, 5 (2006) 221-236

[2] Marolt, D., Knezevic, M., Novakovic, G. V. Bone tissue engineering with human stem cells. Stem Cell Res Ther 1, 10, doi:10.1186/scrt10 (2010) 
[3] Aubin, J. E., Triffitt, J. T. Principles of Bone Biology Vol. 1, Academic Press, 2002, pp. 59-81

[4] Chesnick, I. E. et al. Evaluation of bioreactor-cultivated bone by magnetic resonance microscopy and FTIR microspectroscopy. Bone 40, (2007) 904-912

[5] Potter, K. et al. Non-destructive studies of tissue-engineered phalanges by magnetic resonance microscopy and $\mathrm{X}$-ray microtomography. Bone 38 , (2006) 350-358
[6] Xu, H. et al. Magnetic resonance microscopy for monitoring osteogenesis in tissue-engineered construct in vitro. Physics in medicine and biology 51, (2006) 719-732

[7] Xu, H., Othman, S. F., Magin, R. L. Monitoring tissue engineering using magnetic resonance imaging. Journal of bioscience and bioengineering 106, (2008) 515-527. 